\title{
Multi-Scale Red-Ox Dynamics of Active Metal Catalysts Revealed by a Combination of In Situ Scanning and Transmission Electron Microscopy
}

\author{
Ramzi Farra ${ }^{1}$, Jing Cao ${ }^{1}$, Ali Rinaldi ${ }^{1}$, Zhu-Jun Wang ${ }^{1}$, Elena Willinger ${ }^{1}$, Xing Huang ${ }^{1}$, Mark Greiner ${ }^{2}$, \\ Robert Schlögl $^{1,2}$, Marc-Georg Willinger ${ }^{1,2,3}$ \\ 1. Department of Inorganic Chemistry, Fritz Haber Institute of the Max Planck Society, Berlin, Germany. \\ 2. Max Planck Institute for Chemical Energy Conversion, Muelheim an der Ruhr, Germany. \\ ${ }^{3}$.Department of Colloid Chemistry, Max Planck Institute of Colloids and Interfaces, Potsdam, Germany.
}

The way in which we picture and describe active catalysts is mostly built on indirect observations and conclusions that are drawn on the basis of smart experiments and theoretical modelling. Direct imaging of the atomic arrangement and local compositional analysis is possible by conventional high resolution electron microscopy. However, since the observations are generally performed under vacuum and close to room temperature, the obtained atomistic details concern an equilibrium state that is of limited relevance if the active state of a catalyst is in the focus of the investigation.

In order to observe working catalysts in their active state, we have recently implemented commercially available sample holders for in situ studies under controlled atmosphere and temperature inside a transmission electron microscope.

In order to relate local processes that occur on the nanometre scale with collective processes that involve fast movement of large numbers of atoms, we have furthermore adapted an environmental scanning electron microscope (ESEM) for the investigation of catalytically active surfaces [1].

Using these two instruments, we are now able to cover a pressure range from $10^{-4}$ to $10^{3}$ mbar and a spatial resolution ranging from the $\mathrm{mm}$ to the sub-nm scale.

Both setups are equipped with mass spectrometers (MS) for analysis of the gas-phase composition and detection of catalytic conversion. The latter is important because it helps relating observed dynamics to catalytic function. The influence of the electron beam, which is still required for the observations, is carefully evaluated in each experiment. Observations at different magnification and electron dose rates, beam on/off experiments, as well as cross-comparison of the gas-phase and temperature induced dynamics observed by in situ TEM and in situ SEM help us to identify imaging conditions in which the electron beam does not have a significant influence on the reaction and associated dynamics.

We will present structural dynamics that are observed during oscillatory red-ox reactions on nickel, platinum and copper catalysts (such as Figures 1, 2 and 3).

The ability to directly image the active catalyst and associated morphological changes at high spatial resolution enables us to refine the interpretation of spatially averaged spectroscopic data that was obtained under otherwise similar reaction conditions, for example, during near-ambient-pressure in situ XPS measurements [2].

It will be shown that the ability of observing the adaption of an active surface to changes in the chemical potential of the surrounding gas phase in real-time potentially offers new and direct ways of optimizing catalysts and applied reaction conditions. Under relevant catalytic conditions, the observed metals show rich structural dynamics, oscillatory phase changes, catalyst sintering and splitting. Factors affecting shape, faceting and size of particles will be discussed. On the basis of in situ observations, more realistic models for theoretical description and modelling can be built. Most importantly, movies that are recorded during the reactions will potentially have a significant impact on the way in which active catalysts are described. 
References:

[1] Z.-J. Wang et al., Nature Communications 7, Article number: 13256 (2016).

[2] M. T. Greiner et al., Phys.Chem.Chem.Phys. 17, (2015), p. 25073

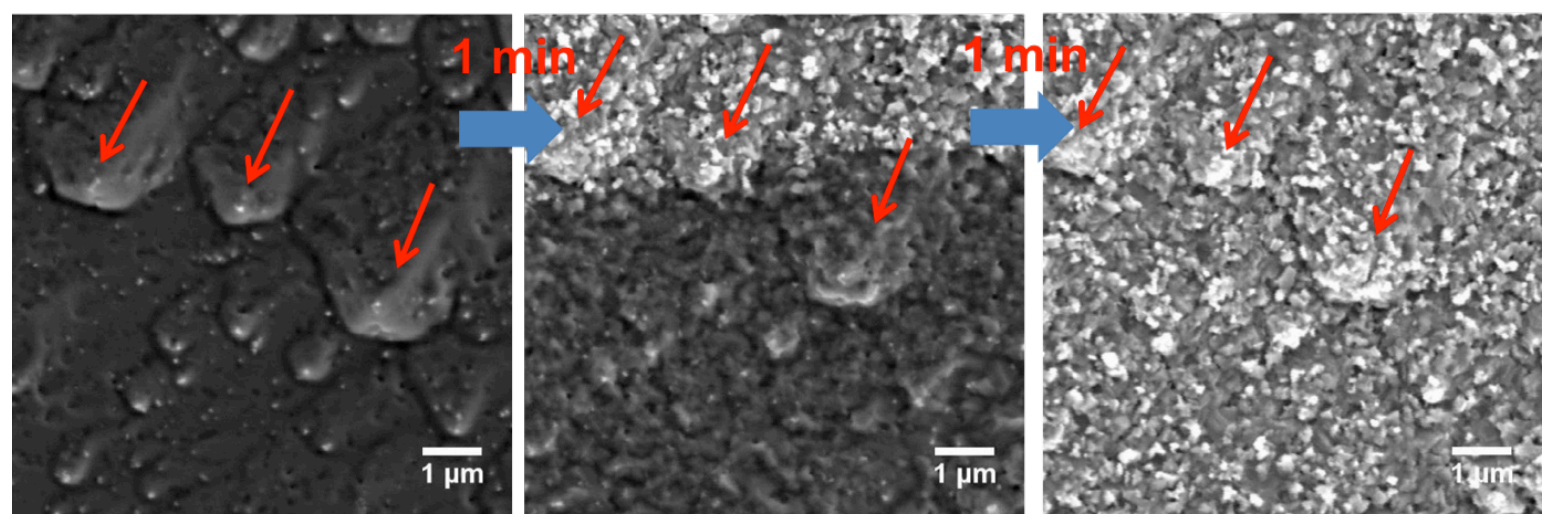

Figure 1. In situ ESEM observations of thermo-kinetic oscillations during hydrogen oxidation on a $\mathrm{Ni}$ foil. The surface shows rich dynamics and periodic changes from an oxide (bright) to a metallic state (dark).
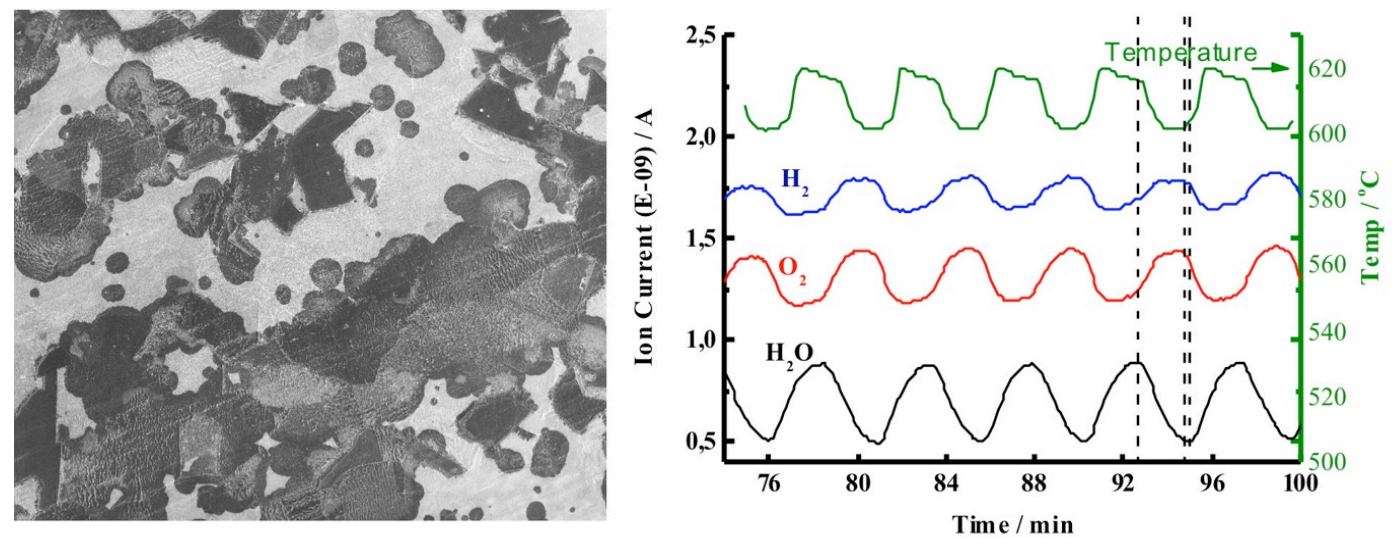

Figure 2. Overview image recorded during in situ observation of redox dynamics of Ni. Changes in contrast due to oxidation and reduction of the Ni are correlated to changes in activity detected in the MS.
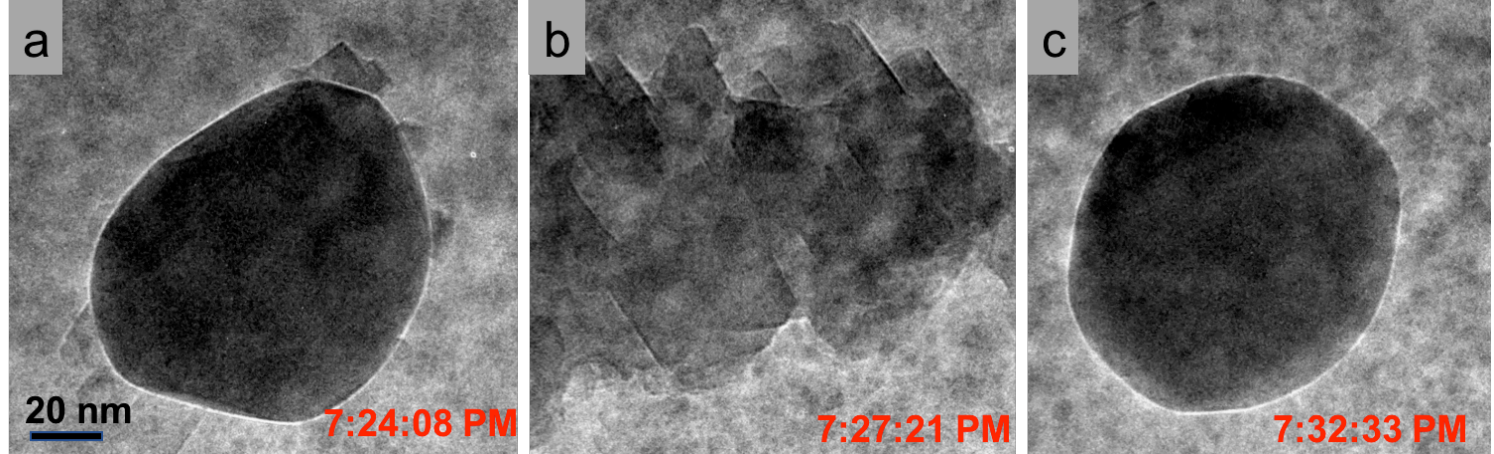

Figure 3. Corresponding in situ TEM observations showing a Ni particle oscillating between metallic (a and c) and oxide state (b) during hydrogen oxidation at $700{ }^{\circ} \mathrm{C}$. 